British Journal of Psychiatry (1991), 159, 724-738

\title{
Correspondence
}

Editor: Ian Pullen

Contents: Conditional probability and sibling sex/ Apparent decrease in schizophrenia/The human brain and political behaviour/'Compulsive' water drinking in psychosis .../... and mentally handicapped people/Negative symptoms of schizophrenia/ Hospital Anxiety and Depression Scale/Neuroleptic Malignant Syndrome/Psychiatrists and citizens/ Frégoli transformed/Promoting the correct use of psychotropic drugs/Affect logic/Exposure therapy for PTSD/Admission rates, detention rates and socioeconomic deprivation/Depression and polycythaemia/What's in a name?/Monozygotic male triplets discordant for psychosis/Chloroquineinduced psychiatric complications/Aggression and multiple sclerosis/Sudden cardiac death and antidepressants/Hair loss associated with fluoxetine.

\section{Conditional probability and sibling sex}

SIR: The letter from Lacey et al (Journal, August $1991,159,291$ ) is a good example of the misuse of Bayesian statistics. Assuming that men and women are equally represented in the population, the probability of a particular sibling being female is $\frac{1}{2}$. This probability is unaffected by my knowledge as to whether other siblings are male or female, older or younger, bulimic or not, or whatever. Specifically, if there are two siblings and one is a female, the probability that the other is female is $\frac{1}{2}$; if I happen to know also that the elder sibling is female, this does not change the situation at all. Any other conclusion is counter-intuitive, as Goodman (Journal, August $1991,159,290)$ neatly illustrates.

Referring to the original paper (Journal, April $1991,158,491-494)$, it can now be seen that Table 2 is incorrect. The purpose of this table is to establish that bulimic females come from all-female sibships more often than expected by chance. The position of the index case within the sibship is clearly irrelevant to this question. Since all the index cases were female, the probability of an all-female sibship of two is simply the probability that the other sibling is female, i.e. $\frac{1}{2}$. Similarly, the probability of an all-female sibship of three is $\left(\frac{1}{2}\right)^{2}$, i.e. $1 / 4$; of four, $\left(\frac{1}{2}\right)^{3}$, i.e. $1 / 8$; of five, $\left(\frac{1}{2}\right)^{4}$, i.e. $1 / 16$. If these figures are used, the expected numbers of such sibships become 40.5, 15.5, 5.5 and 1.5 respectively, which are very similar to the observed numbers of 45, 20,6 and 2. This suggests that Lacey et al have not proved their hypothesis that bulimic girls come from all-female sibships more often than expected by chance.

Paul Bailey

6 Rue Beau Village

F-68730 Blotzheim

France

SIR: It seems clear that Dr Goodman is right in his critique of Lacey $e t a$ l $s$ data on the siblings of patients with bulimia nervosa (Journal, August 1991, $159,290)$. It is the authors who appear to be in error, both in the original article (Journal, April 1991, 158, 491-494) and in their reply (Journal, August 1991, $159,291)$. They argue that by chance alone bulimic women will be less likely to come from same sex sibships than mixed ones and try to prove this using conditional probability theory. They point out that the possibilities for a sibship of two in the population are: MM, MF, FM, FF. If subjects of either sex are selected at random as cases then the probability of coming from any pair is $1 / 4$. The authors are then correct in their statement that the conditional probability of a sibship of two girls given that one is a girl is $1 / 3$ (i.e. $1 / 4 / 3 / 4$ ). However, as their study involved women, prior selection has altered the probabilities of sibling pair occurrence. By including only women, the chance of them coming from an all-female sibship rises from $1 / 4$ to $1 / 2$ (all-female sibships contain two women and so are twice as likely to be selected as male-female ones). In a similar way, assume for simplicity that all the women in the population are taken as coming from sibships of two represented by MM, MF, FM, and FF. Fifty per cent of women would then be in an all-female sibling pair (even though only $25 \%$ of pairs are all female). In other words, women would be expected to have an equal chance of having a brother or a sister. Common sense tells us that this is so and it is not surprising that this is what Lacey et al found in their study. The authors should 